doi: $\underline{10.31211 / \text { interacoes.n36.2019.a4 }}$

\title{
As Tecnologias de Informação e Comunicação no Quotidiano Social da Pessoa Idosa: Breve Revisão Narrativa
}

\author{
José Mendes \\ INTELECTO - Psicologia \& Investigação \\ Instituto de Psicologia Cognitiva, Desenvolvimento Humano e Social (IPCDHS) \\ josemendes78@gmail.com
}

Sumário

As novas tecnologias têm-se demonstrado um importante instrumento na promoção da qualidade de vida e segurança das pessoas idosas. A pesquisa centrou-se maioritariamente em estudos publicados nos últimos cinco anos, com o objetivo de compreender a participação das pessoas idosas numa sociedade mediada pela internet. Explorou-se a relevância das novas tecnologias de informação e comunicação
(TIC) no quotidiano das pessoas idosas e a importância de melhorar a literacia destas num envelhecimento construtivo. As TIC quando adaptadas às necessidades da pessoa idosa, podem revelar-se uma importante ferramenta na participação de movimentos sociais, promovendo a pessoa idosa numa sociedade em constante transformação tecnológica.

Palavras-chave: Idoso, informação, internet, novas tecnologias.

\section{Information and Communication Technology in Day Life of Elder People: a Brief Review Narrative}

\begin{abstract}
New technology was demonstrated an important tool to promote the security and quality of life in older people. This research was centred in publish studies in the last five years. The objective is to understand their participation in society through the internet. New information and communication technology (ICT) were

explored in the older people day life and the importance to improve literacy an aging constructive. ICTs adapted to the needs of the elderly can prove to be an important tool in the participation of social movements, promoting older people in a society in constant technological transformation.
\end{abstract}

Keywords: Older adults, information, internet, new technology. 


\section{INTRODUÇÃ̃O}

A população mundial está cada vez mais envelhecida, prevendo-se que os idosos ultrapassem os grupos etários de crianças e adolescentes num futuro próximo (Amorim, Sampaio, Carvalho, \& Vilaça, 2018; Carmo \& Zazzetta, 2016; Moura, Gosling, Christino, \& Macedo, 2017; Santos, Feitosa, \& Silva, 2016). Neste sentido, o envelhecimento ativo insere-se de forma muito gradual nas novas tecnologias, especificamente às TIC (Santos et al., 2018).

A tecnologia e a ciência estão presentes no quotidiano de todos os indivíduos, desempenhando um papel determinante na transformação e modificação da economia mundial (Dias, 2005). A evolução social e tecnológica conduz o indivíduo a uma aprendizagem e educação formal ou informal (Ribeiro \& Medeiros, 2016).

Tavares et al. (2017) referem que a dimensão social é percecionada como uma das dimensões que contribuem para um envelhecimento saudável. As novas formas de estar e viver dos idosos distinguem-se no processo de envelhecimento, conhecimento de si e participação de atividades cívicas, existindo a necessidade de fornecer o acesso destes às TIC (Ribeiro \& Medeiros, 2016).

A discussão sobre os impactos da utilização das TIC são diariamente difundidas pelos meios de comunicação social (i.e., televisão, jornais), no entanto, é na faixa etária dos adolescentes que se concentram a maioria das questões sobre os benefícios e malefícios das TIC no desenvolvimento cognitivo, afetivo e social dos mesmos (Silva \& Silva, 2017). Medeiros (2016) defende que, perante o aumento da população idosa, existe uma necessidade de reajustamento social, devendo valorizar-se o papel das pessoas idosas e o seu contributo como cidadãos. Salienta-se, assim, a variedade de fatores que influenciam a heterogeneidade no processo de envelhecimento (Katzenstein, Schwartz, \& Almeida, 2012), reconhecendo-se a falta de investigação que foque a utilização e dependência das TIC por pessoas idosas (Felizmino \& Barbosa, 2018).

Até à segunda metade do século XX, o envelhecimento centrava-se na autonomia do indivíduo (Novo, 2016), envolvendo a componente biológica, social e psicológica (Dias, 2012), onde o processo de envelhecimento, apesar de idêntico em termos biológicos, não deve ser perspetivado de forma comum (Neves, 2016), pois a reconstrução da identidade do envelhecimento é um processo idiossincrásico (Novo, 2016). Medeiros (2016) defende o envelhecimento como um constructo complexo que exige uma atenção multidisciplinar, no intuito de promover a igualdade, a inclusão e o bem-estar psicológico da pessoa idosa. 
As Tecnologias de Informação e Comunicação no Quotidiano Social da Pessoa Idosa: Breve Revisão Narrativa

A pessoa idosa, devido ao aumento da expetativa de vida, procura acompanhar a evolução das TIC (Pereira \& Neves, 2011; Verona, Cunha, Pimenta, \& Buriti, 2006), existindo, na maioria das vezes, dificuldade por partes das pessoas idosas em compreender e integrar esta nova realidade, excluindo-os de conhecer verdadeiramente a era digital (Pereira \& Neves, 2011). Uma das razões pelas quais as pessoas idosas percecionam as TIC como difíceis de utilizar (Raymundo \& Santana, 2015), pode dever-se ao facto de estas se considerarem redes de comunicação altamente desenvolvidas ou ser resultado do próprio processo de envelhecimento (i.e., visão, motricidade) (Farias, Vitor, Lins, \& Pedroza Filho, 2015).

O progresso tecnológico veio privar o idoso do seu papel e prestígio social, no entanto, a introdução das TIC apresentam novas possibilidades e limites na integração da pessoa idosa na sociedade (Dias, 2012). Petersen, Kalempa e Pykosz (2013) defendem a necessidade de o indivíduo envelhecer sem perder conexão com o mundo cada vez mais digital, onde a pessoa idosa pode ocupar o seu tempo e manter um conhecimento continuo com recurso às TIC, conservando assim a sua saúde mental e manter uma rede de apoio social ativa.

Estudos têm demonstrado a importância das TIC como ferramentas relevantes na realização das atividades instrumentais na vida diária das pessoas idosas de forma a capacitar a sua autonomia e independência (Carmo \& Zazzetta, 2016; Souza \& Silva, 2016), capacitando-as na prevenção de acidentes domésticos (Campos et al., 2017). As TIC desenvolvem competências a nível de alfabetização e fluência digital (Machado, Grande, Behar, \& Luna, 2016) e contribuem para uma saúde preventiva (Santos et al., 2018). Araújo et al. (2017) revelam que as TIC são um avanço da ciência que favorece os diversos serviços de saúde à pessoa idosa.

Apesar de a utilização das TIC pelas pessoas idosas se considerar uma "tecnologia assistiva" (Gil, 2014), Felizmino e Barbosa (2018) mencionam uma forte evolução da utilização das TIC por pessoas idosas nos últimos 10 anos, recorrendo às tecnologias como agente facilitador do quotidiano (i.e., compras, conhecer pessoas), remodelar a sua representação social e otimizar a saída da ociosidade e sedentarismo. As TIC proporcionam às pessoas idosas um estilo de vida saudável, aumentam a sua rede social, contribuem para o bem-estar, melhoram os níveis de independência (Lee, Han, \& Chung, 2014) e proporcionam uma infinidade de possibilidades (Carmo \& Zazzetta, 2016).

Um estudo de Chopik (2016) revela que são poucos os estudos que avaliam as associações entre a utilização das TIC por razões sociais e a saúde física/psicológica 
entre as pessoas idosas. Perante a multiplicidade das TIC na interação, divulgação de mecanismos de mobilização e movimentos sociais, sobretudo pelos jovens (Queiroz, 2017), o desenvolvimento de novas formas de decisão política devido à mediatização da internet (Hayashi, Rigolin, Rothberg, \& Hayashi, 2011) e a necessidade da construção partilhada de políticas públicas (Rothberg, 2010), revela-se pertinente compreender qual uso das TIC pelas pessoas idosas na participação em movimentos sociais.

\section{MÉTODO}

Procedeu-se a uma filtragem de publicações elegíveis à revisão de literatura, tendo em consideração as seguintes etapas: i) definição de uma questão de investigação e critérios de elegibilidade; ii) pesquisa de artigos em base de dados (Google Scholar e Ebsco) com aplicação dos operadores booleanos AND e NOT, tendo em considera-

ção as palavras chave: idoso; informação; internet; novas tecnologias. Adotaram-se os critérios de inclusão: i) uma relação entre as palavras chave; ii) as publicações pertencerem ao espaço lusófono e ibero-americano na língua portuguesa, francesa, espanhola e inglesa e iii) a publicação ser de cariz científico em texto completo entre os anos 2014 e 2019.

\section{RESULTADOS}

A filtragem das publicações encontradas nas bases de dados permitiu identificar 24 publicações em texto completo.

\section{As TIC nas políticas de inclusão da pessoa idosa}

A inclusão digital, além de contribuir para a inserção social, colabora com um envelhecimento ativo (Souza \& Silva, 2016). O acesso equitativo e onipresente permitem que a população alcance o uso das TIC, em que a utilização da internet é propiciada pelo otimismo e proficiência, sendo o fator vulnerabilidade o fator de maior peso na inibição da adoção das TIC pelas pessoas idosas (Farias et al., 2015). No entanto, Raymundo e Santana (2015) observam dois processos contrários: um considerado pelo rápido desenvolvimento das TIC em países desenvolvidos e em 
As Tecnologias de Informação e Comunicação no Quotidiano Social da Pessoa Idosa: Breve Revisão Narrativa

desenvolvimento e o outro pelo rápido envelhecimento da população de países em crescimento.

As pessoas idosas encontram-se no extremo oposto no que se refere à utilização das TIC quanto às gerações mais novas. Os avanços tecnológicos limitam muitas vezes as pessoas idosas de lidar com elementos básicos (e.g., eletrodomésticos mais sofisticados) no seu quotidiano (Santos, Feitosa, \& Silva, 2016). A integração das pessoas idosas numa sociedade cada vez mais tecnológica, leva a uma necessidade de estes aprenderem a usar as TIC de forma a se relacionarem com a informação, comunicação digital, criatividade e atividades de lazer (Calvo, Elorriaga, Arruarte, Larrañaga, \& Gutiérrez, 2017).

As dificuldades sentidas no uso das TIC podem estar relacionadas com os equipamentos digitais e a pessoa idosa, existindo uma necessidade de aceitação das TIC para que estas se possam inserir no quotidiano do indivíduo (Raymundo \& Santana, 2015). O processo educacional das TIC para as pessoas idosas pode considerar-se complexo (Calvo et al., 2017), existindo a necessidade de criar propósitos de aprendizagem que facilitem o processo de ensino/aprendizagem por parte das pessoas idosas, permitindo o acompanhamento da evolução das novas tecnologias (Machado, Grande, Behar, \& Luna, 2016).

O surgimento das TIC pode gerar preocupações e desconforto nas pessoas idosas, devido ao receio de estas poderem ser excluídas por não saberem utilizar tais tecnologias (Silva, 2016). Muitas pessoas idosas ainda estão digitalmente excluídas devido aos níveis de escolaridade e baixos rendimentos, revelando-se pertinente um maior investimento de estratégias que promovam a inclusão digital (Carmo \& Zazzetta, 2016; Krug, Xavier, \& D’Orsi, 2018)

\section{As TIC na promoção de saúde da pessoa idosa}

As TIC consideram-se importantes no quotidiano das pessoas idosas por lhes proporcionar maior autonomia, bem-estar e interação social, pois a conexão e comunicação com o mundo promovem a qualidade de vida das pessoas idosas (Skura, Velho, Francisco, Faria, \& Macuch, 2013). Antunes e Abreu (2017) revelam que as TIC, além de proporcionam níveis de bem-estar físico, psicológico e relacionamento interpessoal, permitem a aquisição de novas aprendizagens.

As perdas biológicas na idade avançada obrigam os idosos a selecionar atividades mais significativas, de forma a otimizar as capacidades existentes por meio de práticas e novas tecnologias (OMS, 2015). Souza e Silva (2016) dão como exemplo as 
aplicações para smartphones se considerarem importantes ferramentas no apoio das atividades instrumentais da vida diária das pessoas idosas. Estas aplicações permitem maior independência e sentimentos de segurança para o idoso e respetivos familiares e cuidadores.

Aspetos inerentes ao envelhecimento, podem inserir-se no quotidiano das pessoas idosas através das TIC. A elaboração de aplicações que promovam a prática de exercício selecionados para a postura, equilíbrio e marcha podem ser aliados na promoção de saúde e prevenção de declínio físico (Santos et al., 2018). Por exemplo, um programa de prevenção de quedas no domicílio com recurso às TIC, pode capacitar as pessoas idosas de maior conhecimento sobre as causas e possíveis acidentes domiciliares, despertando o interesse pelas TIC (Campos et al., 2017).

O envolvimento das TIC no envelhecimento ativo, tem produzido efeitos positivos na perceção que as pessoas idosas têm da sua qualidade de vida e aceitação social (Ferreira, Torres, Mealha, \& Veloso, 2015). As TIC, através de aplicativos específicos, permitem e facilitam a comunicação entre as pessoas idosas e o sistema de saúde (e.g., médicos), transmitindo remotamente os sinais vitais e outras informações relevantes que possam colocar em causa o bem-estar e a qualidade de vida da pessoa idosa (Czaja, 2015).

Uma recente revisão de literatura verificou que os aplicativos desenvolvidos para o auxílio nos cuidados e na saúde da pessoa idosa influenciam a exploração das TIC por parte dos idosos (Amorim et al., 2018). Santos et al. (2018) reforçam a necessidade de delinear programas que relacionem o uso de tecnologias e a atividade física ao processo de envelhecimento.

$\mathrm{Na}$ sociedade atual, as TIC e a Saúde estão presentes no quotidiano de todos os indivíduos, principalmente nos profissionais de saúde (Schmeil, 2013), agilizam a informação (Santos et al., 2017) e auxiliam a gestão em saúde da pessoa idosa (Romero et al., 2018). No entanto, a pouca exploração das TIC pelos profissionais de saúde (Mota, Torres, Guimarães, Marinho, \& Araújo, 2018) e a falta de diretrizes políticas que auxiliam estes profissionais a melhor utilizarem estas tecnologias, criam barreiras no planeamento e acompanhamento de programas de saúde do idoso (Romero et al., 2018).

\section{As TIC e a participação social da pessoa idosa}

A difusão de informação por meio da internet, é considerada como a revolução digital que passou dos meios de comunicação social para formas de difusão de in- 
As Tecnologias de Informação e Comunicação no Quotidiano Social da Pessoa Idosa: Breve Revisão Narrativa

formação mais individualizada (Queiroz, 2017). A partilha de experiências de vida pelas pessoas idosas permite uma produção de conhecimento e desenvolve maior autoestima (Silva, 2016). O consumo cultural pode ser uma oportunidade de a pessoa idosa ter uma participação mais ativa na sociedade (Ferrigno, 2016). O aumento do conhecimento por meio da inclusão digital permite maior comunicação e interação, promovendo a participação em grupos de aprendizagem que propiciem novos hábitos de vida durante o envelhecimento (Carmo \& Zazzetta, 2016).

A participação social é reconhecida como um indicador de envelhecimento ativo, influenciando a saúde e o bem-estar das pessoas idosas (Rebellato \& Hayashi, 2014). Pinto e Neri (2017) apontam a necessidade de uma maior reflexão da idealização política que coloca em prática a participação social no envelhecimento ativo. Na opinião de Silva (2016), as pessoas idosas percecionam a necessidade de uma convivência entre gerações onde as responsabilidades e a definição dos papéis sociais a serem desempenhados, influenciam a construção de uma cultura de civilidade.

A emergência das TIC tem promovido o envelhecimento ativo dotando os indivíduos de competências que contribuem para o menor isolamento, maior relacionamento interpessoal e novas perspetivas de vida (Antunes \& Abreu, 2017). Estes autores defendem ainda que as pessoas idosas, por meio das TIC, assumem papéis ativos no seu processo de desenvolvimento. A participação social está intimamente ligada à cultura, crenças, hábitos e recursos disponíveis na comunidade (Pinto \& Neri, 2017), salientando-se assim a importância de uma intervenção educativa, por exemplo, a implantação das TIC nas universidades seniores.

O comportamento social das pessoas idosas é influenciado por experiências vividas, existindo a necessidade de estas pessoas estabelecerem vínculos sociais precoces (Pinto \& Neri, 2017). Puerta (2016) sugere um envelhecimento construtivo através de experiências educativas, que proporcionem uma aprendizagem contínua entre os diferentes níveis educativos. Este autor destaca que as universidades seniores, devem possuir características especificas e diferenciadas agrupando diferentes temáticas, proporcionando na visão de Ferrigno (2016), uma mudança na representação social das pessoas idosas, pelo interesse que estes mantêm em continuar a aprender.

\section{Discussão e considerações finais}

Apurou-se com este trabalho que a maioria das investigações centradas na utilização das TIC pela pessoa idosa se centra no espaço lusófono, tendo como principais temáticas a importância da inclusão desta faixa etária para a utilização das TIC e o 
seu impacto na qualidade de vida na pessoa idosa. As investigações realizadas no Brasil revelam, ao longo dos últimos cinco anos, uma preocupação com as políticas de proteção da pessoa idosa. Por sua vez, em Portugal, defende-se o "(RE)Pensar as pessoas idosas no século XXI” por meio de um pensamento articulado, participativo e construtivo (Medeiros, 2016).

Faz-se notar que a investigação na avaliação das TIC pelas pessoas idosas se concentra mais nas vantagens voltadas para o cuidado e capacidades funcionais sendo mais negligenciados aspetos como as potencialidades da utilização das TIC no quotidiano da pessoa idosa (i.e., participação cívica). Apesar de a popularização da internet ter desenvolvido novas formas de decisão política e implementação de mecanismos de consulta pública, não se encontraram artigos que manifestem a participação das pessoas idosas em movimentos sociais com recurso às TIC.

Tendo em consideração o modelo de aceitação de tecnologia, em especial, a perceção da facilidade do uso e a perceção da utilidade, comumente aplicado no estudo de Raymundo e Santana (2015), as pesquisas futuras devem concentrar-se em explorar a participação social das pessoas idosas por meio das TIC. Apesar da evolução tecnológica incorporada no quotidiano das pessoas idosas resultar em novos processos de exclusão (Silva, 2016), deve ter-se em consideração as desigualdades sociais e os diferentes níveis de escolaridade (Krug et al., 2018).

O surgimento da internet implementou inúmeras formas de comunicar (e.g., sites, redes sociais, blogs), mas são maioritariamente acedidas pelos jovens (Queiroz, 2017). As políticas de inclusão das TIC no quotidiano das pessoas idosas parecem estar mais direcionadas na capacitação desta tecnologia (Araújo et al., 2017; Krug et al., 2018; Santos et al., 2018) e negligenciam a potencialidade destas numa participação social ativa.

O envolvimento das pessoas idosas em atividades sociais influencia a saúde física e mental, colaborando no bem-estar global (Pinto \& Neri, 2017). No entanto, a construção de uma democracia em rede ainda está longe de se tornar uma realidade por meio da criação de tecnologias cívicas criadas pela sociedade civil (Filho \& Martins, 2017). Nesse sentido, é emergente desenvolver aplicações por meio das TIC, que permitam às pessoas idosas com necessidades especiais (e.g., questões ergonómicas) o direito as utilizar (Calvo et al., 2017), pois as dificuldades na utilização das TIC traz o desinteresse das pessoas idosas em utilizarem estas ferramentas no seu quotidiano (Batista, Souza, Schwartz, Exner, \& Almeida, 2015). 
As Tecnologias de Informação e Comunicação no Quotidiano Social da Pessoa Idosa: Breve Revisão Narrativa

Em conclusão, o presente estudo é comprovado por Medeiros (2016) onde se refere a necessidade de a investigação sobre as pessoas idosas se centrar na construção de um pensamento que otimize as relações intergeracionais, focando a capacidade da pessoa idosa na construção social.

\section{REFERÊNCIAS}

Amorim, D. N. P., Sampaio, L. V. P., Carvalho, G. A., \& Vilaça, K. H. C. (2018). Aplicativos móveis para a saúde e o cuidado de idosos. Revista Eletrônica de Comunicação, Informação e Inovação em Saúde, 12(1). doi:10.29397/reciis. $\underline{\mathrm{v} 12 \mathrm{i} 1.1365}$

Antunes, M. C., \& Abreu, V. (2017). As novas tecnologias na promoção do envelhecimento bem-sucedido. Ensino e Tecnologia em Revista, 1(1), 3-15. Retrived from https://periodicos.utfpr.edu.br/etr/article/view/5885

Araújo, S. N. M., Santiago, R. F., Barbosa, C. N. S., Figueiredo, M. L. F., Rangel, E. M. L., \& Nery, I. S. (2017). Tecnologias voltadas para o cuidado ao idoso em serviços de saúde: uma revisão integrativa. Enfermería Global, 16(2), 562. doi:10.6018/ eglobal.16.2.247241

Batista, M. P. P., Souza, F. G., Schwartz, G., Exner, C., \& Almeida, M. H. M. (2015). Utilização no cotidiano de tecnologias da informação e comunicação por idosos participantes da Universidade Aberta da Terceira Idade da Universidade de São Paulo. Revista Kairós Gerontologia, 18(4), 405-426. Retrived from https://revistas.pucsp.br/kairos/article/view/30257/20930

Calvo, I., Elorriaga, J. A., Arruarte, A., Larrañaga, M., \& Gutiérrez, J. (2017). Introducing computer-based concept mapping to older adults. Educational Gerontology, 43(8), 404-416. doi:10.1080/03601277.2017.1309635

Carmo, E. G., \& Zazzetta, M. S. (2016). Envelhecimento, novas tecnologias e aposentadoria. Em J. L. R. Costa, A. M. M. R. Costa, \& G. F. Junior (Eds.), O que vamos fazer depois do trabalho? Reflexões sobre a preparação para aposentadoria (pp. 93-101). doi:10.7476/9788579837630 
Dias, I. (2012). O uso das tecnologias digitais entre os seniores: motivações e interesses. Sociologia, Problemas e Práticas, 2012(68). doi:10.7458/SPP201268693

Dias, M. R. (2005). Serão os mass media estrategas de saúde? Lisboa: Climepsi Editores.

Felizmino, T. D. O., \& Barbosa, R. B. (2018). Idosos e dependência de internet: uma revisão bibliográfica. Revista Psicologia, Diversidade e Saúde, 7(1), 139. doi:10.17267/2317-3394rpds.v7i1.1669

Ferrigno, J. C. (2016). O cidadão idoso: consumidor e produtor cultural. Em A. O. Alcântara, A. A. Camarano, \& K. C. Giacomin (Eds.), Política nacional do idoso: velhas e novas questões (pp. 343-358). Rio de Janeiro: Ipea.

Filho, H. C. P. P., \& Martins, R. A. P. (2017). Um impasse e três saídas para um novo ciclo de tecnologias cívicas livres de participação social. Em J. P. Mehl \& S. P. Silva (Eds.), Cultura Digital, internet e apropriações políticas: Experiências, desafios e horizontes (pp. 193-202). doi:10.24328/2017/5473.004/11

Gil, H. T. (2014). Os cidadãos mais idosos (65+anos) do concelho de Castelo Branco na utilização das TIC, e-Saúde e e-Governo Local. Instituto Superior de Ciências Sociais e Políticas. Retrived fom: https://repositorio.ipcb.pt/bitstream/10400.11/2463/1/Texto\%20completo\%20ccapa.pdf

Hayashi, Ma. C. P., Rigolin, C. C. D., Rothberg, D., \& Hayashi, C. R. M. (2011). Democracia digital, participação e disseminação do conhecimento. Em M. C. Hayashi, C. M. Sousa, \& D. Rothberg (Eds.), Apropriação social da ciência e da tecnologia: contribuições para uma agenda (pp. 191-217). Campina Grande: EDUEPB.

Katzenstein, T., Schwartz, G., \& Almeida, M. H. M. (2012). Reflexões sobre aproximação de idosos a tecnologias de informação e comunicação a partir dos arquétipos Senex ${ }^{\star}$ e Puer. Revista Kairós Gerontologia, 15(3), 203-218. Retrived from https://revistas.pucsp.br/index.php/kairos/article/view/9883

Krug, R. D. R., Xavier, A. J., \& D’Orsi, E. (2018). Fatores associados à manutenção do uso da internet, estudo longitudinal EpiFloripa Idoso. Revista de Saúde Pública, 52, 37. doi:10.11606/S1518-8787.2018052000216 
As Tecnologias de Informação e Comunicação no Quotidiano Social da Pessoa Idosa: Breve Revisão Narrativa

Lee, E., Han, S., \& Chung, Y. (2014). Internet Use of Consumers Aged 40 and Over: Factors That Influence Full Adoption. Social Behavior and Personality: An International Journal, 42(9), 1563-1574. doi:10.2224/sbp.2014.42.9.1563

Machado, L. R., Grande, T. P. F., Behar, P. A., \& Luna, F. M. R. (2016). Mapeamento de competências digitais: a inclusão social dos idosos. ETD - Educação Temática Digital, 18(4), 903. doi:10.20396/etd.v18i4.8644207

Medeiros, T. (2016). (Re)Pensar as pessoas idosas no século XXI. Ponta Delgada: Letras Lavadas.

Mota, D. N., Torres, R. augusto M., Guimarães, J. M. X., Marinho, M. N. A. S. B., \& Araújo, A. F. (2018). Tecnologias da informação e comunicação: influências no trabalho da estratégia Saúde da Família. Journal of Health Informatics, 10(2), 45-49. Retrived from http://www.jhi-sbis.saude.ws/ojs-jhi/index.php/jhi-sbis/ article/viewFile/563/330

Moura, A. C., Gosling, M. D. S., Christino, J. M. M., \& Macedo, S. B. (2017). Aceitação e uso da tecnologia para escolha de destinos turísticos por pessoas da terceira idade: um estudo usando a UTAUT2. Revista Brasileira de Pesquisa em Turismo, 11(2), 239. doi:10.7784/rbtur.v11i2.1277

Neves, M. C. P. (2016). No nosso segundo aniversário, o envelhecimento... Em T. Medeiros (Eds.), (Re)Pensar as pessoas idosas no século XXI (pp. 31-45). Ponta Delgada: Letras Lavadas.

Novo, R. F. (2016). Desenvolvimento e envelhecimento: velhas e novas metáforas. Em T. Medeiros (Eds.), (Re)Pensar as pessoas idosas no século XXI (pp. 47-70). Ponta Delgada: Letras Lavadas.

Pinto, J. M., \& Neri, A. L. (2017). Trajectories of social participation in old age: a systematic literature review. Revista Brasileira de Geriatria e Gerontologia, 20(2), 259-272. doi:10.1590/1981-22562017020.160077

Puerta, J. A. (2016). Las Universidades para mayores en España: un espacio para el envejecimiento construtivo. Em T. Medeiros (Eds.), (RE)Pensar as pessoas idosas no século XXI (pp. 313-328). Ponta Delgada: Letras Lavadas.

Queiroz, E. F. C. (2017). Ciberativismo: a nova ferramenta dos movimentos sociais. Panorama, 7(1), 2. doi:10.18224/pan.v7i1.5574 
Raymundo, T. M., \& Santana, C. da S. (2015). Elderly perception about the new technologies. Inteligencia Artificial, 18(55), 12. doi:10.4114/intartif.vol18iss55pp12-25

Rebellato, C., \& Hayashi, M. C. (2014). Participação social do idoso: estudo bibliométrico da produção científica recente (2010-2013). Revista Eletrônica de Comunicação, Informação e Inovação em Saúde, 8(3), 264-287. Retrived from https://www.reciis.icict.fiocruz.br/index.php/reciis/article/view/444

Ribeiro, E., \& Medeiros, T. (2016). Educação sénior: a voz de estudantes adultos em idade avançada. Em T. Medeiros (Eds), (Re)Pensar as pessoas idosas no século XXI (pp. 241-264). Ponta Delgada: Letras Lavadas.

Romero, D. E., Castanheira, D., Marques, A. P., Muzy, J., Sabbadini, L., \& Silva, R. S. (2018). Metodologia integrada de acompanhamento de políticas públicas e situação de saúde: o SISAP-Idoso. Ciência \& Saúde Coletiva, 23(8), 2641-2650. doi: $\underline{10.1590 / 1413-81232018238.10302016}$

Rothberg, D. (2010). Contribuições a uma teoria da democracia digital como suporte à formulação de políticas públicas. Revista CTS, 14(5), 87-105. Retrived from http://www.revistacts.net/volumen-5-numero-14/90-articulos/311-contribuicoes-a-uma-teoria-da-democracia-digital-como-suporte-a-formulacao-de-politicas-publicas

Santos, C. M. V. T., Andrade, J. A., Amorim, A. do C., Garcia, P. A., Carvalho, G. A., \& Vilaça, K. H. C. (2018). Application on mobile platform "Idoso Ativo" (Active Aging): exercises for lower limbs combining technology and health. Fisioterapia em Movimento, 31. doi:10.1590/1980-5918.031.ao17

Santos, D. B., Feitosa, E. T., \& Silva, R. O. (2016). O uso de tecnologias pela população idosa brasileira. Tecnologias em Projeção, 7(2), 80-87. Retrived from http:// revista.faculdadeprojecao.edu.br/index.php/Projecao4/article/view/697

Santos, A. F., Fonseca S. D., Araujo, L. L., Procópio, C. S. D., Lopes, É. A. S., Lima, A. M. L., ... Matta-Machado, A. T. (2017). Incorporação de Tecnologias de Informação e Comunicação e qualidade na atenção básica em saúde no Brasil. Cadernos de Saúde Pública, 33(5). doi:10.1590/0102-311x00172815 
As Tecnologias de Informação e Comunicação no Quotidiano Social da Pessoa Idosa: Breve Revisão Narrativa

Schmeil, M. A. (2013). Saúde e Tecnologia da Informação e Comunicação. Fisioterapia em Movimento, 26(3), 477-478. doi:10.1590/S0103-51502013000300001

Silva, M. R. F. (2016). Envelhecimento e proteção social: aproximações entre Brasil, América Latina e Portugal. Serviço Social \& Sociedade, 126, 215-234. doi:10.1590/0101-6628.066

Silva, M. C. (2016). As tecnologias de comunicação na memória dos idosos. Serviço Social \& Sociedade, (126), 379-389. doi:10.1590/0101-6628.074

Silva, T. O., \& Silva, L. T. G. (2017). Os impactos sociais, cognitivos e afetivos sobre a geração de adolescentes conetados às tecnologias digitais. Revista Psicopedagogia, 34(103), 87-97. Retrived from http://pepsic.bvsalud.org/scielo.php?script=sci arttext\&pid=S0103-84862017000100009

Tavares, R. E., Jesus, M. C. P., Machado, D. R., Braga, V. A. S., Tocantins, F. R., \& Merighi, M. A. B. (2017). Envelhecimento saudável na perspectiva de idosos: uma revisão integrativa. Revista Brasileira de Geriatria e Gerontologia, 20(6), 878-889. doi: 10.1590/1981-22562017020.170091 